TENDENCIAS

Revista de la Facultad de Ciencias

Económicas y Administrativas.

Universidad de Nariño

ISSN-E 2539-0554

Vol. XXIII No. 1 - 1er Semestre 2022

Enero - Junio - Páginas 139-166

\title{
PROSPECTIVA ESTRATÉGICA DEL SUBSECTOR COMERCIO DEL MUNICIPIO DE IPIALES - COLOMBIA, HACIA EL AÑO 2030
}

STRATEGIC FORESIGHT OF THE COMMERCE SUBSECTOR OF THE MUNICIPALITY OF IPIALES - COLOMBIA, TOWARDS THE YEAR 2030

PREVISÃO ESTRATÉGICA DO SUBSECTOR DO COMÉRCIO DO MUNICÍPIO DE IPIALES - COLÔMBIA, PARA O ANO 2030

\author{
Eduardo D. Chalapud
}

Magíster en Administración y Competitividad, Universidad Mariana. Docente - Investigador Corporación Unificada Nacional de Educación Superior CUN. ORCiD: 0000-0003-0723-2149. E-mail: eduardo_chalapud@cun.edu.co, Colombia.

Recibido: 23 de junio de 2021

Aprobado: 19 de octubre de 2021

DOI: https://doi.org/10.22267/rtend.222301.186

\section{Resumen}

En el presente artículo se realiza un estudio prospectivo del subsector comercio al por mayor y menor en la zona de frontera colombo - ecuatoriana, específicamente en el municipio de Ipiales hacia el año 2030. Su importancia en la dinámica económica la posiciona como la principal actividad y fuente generadora de empleo, por ello es necesario su fortalecimiento examinando la influencia de diversos factores indispensables en el presente y su desarrollo en el futuro. Se examinan los factores de incidencia que son convertidos a variables clave por expertos utilizando el método Mic Mac; mediante el análisis de actores se constata las relaciones de convergencia y 
divergencia entre ellos y se realiza los escenarios utilizando el análisis Morfológico. Por último, se clasifican estrategias inmediatas, reto y menos urgente a realizar que, con su implementación facilita el cumplimiento del escenario apuesta. Se busca con ello rediseñar la cadena de valor y de suministro, aprovechar la infraestructura vial, participar directamente con los centros de producción, fortalecer la asociatividad y el Clúster comercial de la frontera.

Palabras clave: actividad económica regional; competitividad; organización de la producción; servicios; transportes.

JEL: R11; H11; L23; L8; L91

\begin{abstract}
This article is a prospective study of the wholesale and retail trade subsector in the ColombianEcuadorian border zone, specifically in the municipality of Ipiales, towards the year 2030. Its importance in the economic dynamics positions it as the main activity and source of employment generation; therefore, it is necessary to strengthen it by examining the influence of various indispensable factors in the present and its development in the future. Incidence factors are examined and converted into key variables by experts using the Mic Mac method; through the analysis of actors, the relations of convergence and divergence between them are ascertained and scenarios are developed using Morphological analysis. Finally, immediate, challenge and less urgent strategies are classified to be carried out, which, with their implementation, facilitate the fulfillment of the bet scenario. The aim is to redesign the value and supply chain, take advantage of the road infrastructure, participate directly with the production centers, strengthen associativity and the border trade cluster.
\end{abstract}

Keywords: regional economic activity; competitiveness; organization of production; services; transportation.

JEL: R11; H11; L23; L8; L91

\title{
Resumo
}

Este artigo realiza um estudo prospectivo do subsector do comércio grossista e retalhista na zona fronteiriça colombiano-equatoriana, especificamente no município de Ipiales, por volta do ano 
2030. A sua importância na dinâmica económica posiciona-o como a principal actividade e fonte de geração de emprego, razão pela qual é necessário reforçá-lo examinando a influência de vários factores indispensáveis no presente e o seu desenvolvimento no futuro. Os factores de incidência são examinados e convertidos em variáveis-chave por especialistas utilizando o método Mac Miro; através da análise dos actores, as relações de convergência e divergência entre eles são averiguadas e os cenários são levados a cabo utilizando a análise Morfológica. Finalmente, são classificadas estratégias imediatas, de desafio e menos urgentes, que, com a sua implementação, facilitam o cumprimento do cenário. $\mathrm{O}$ objectivo é redesenhar a cadeia de valor e fornecimento, aproveitar as infra-estruturas rodoviárias, participar directamente com os centros de produção, reforçar a associatividade e o agrupamento do comércio fronteiriço.

Palavras-chave: actividade económica regional; competitividade; organização da produção; serviços; transportes.

JEL: R11; H11; L23; L8; L91

\section{Introducción}

La importancia de la frontera colombo - ecuatoriana desde un enfoque histórico, ha demarcado relaciones comerciales dinámicas entre las ciudades fronterizas de Ipiales -Colombia- y Tulcán Ecuador- (Mosquera y Forero, 2017). Estas ciudades, consideradas interdependientes, se encuentran separadas por el Puente Internacional de Rumichaca, considerado el paso fronterizo de mayor importancia en el suroccidente colombiano y que sirve de garante para el intercambio comercial, tradicional e histórico entre los dos países (Grabendorff, 2017). Las relaciones comerciales dinámicas han dado pie para la preservación de prácticas sociales y formas de vida similares entre las comunidades que habitan la zona (Solarte et al., 2020).

Es de interés analizar el subsector comercio al por mayor y menor para determinar los escenarios de cambio que permitan su evolución y contribuya al desarrollo de la región entrelazando las diferentes variables, actores y estrategias que promuevan un alto comportamiento mediante un estudio prospectivo estratégico de la actividad más importante en la zona de frontera. 
En el presente estudio se relata la dinámica del subsector comercio, su influencia e importancia en la economía de la región, se utilizan fuentes secundarias especializadas, teniendo en cuenta la restricción de estudios e indicadores económicos continuos. Además, se realiza el análisis estructural del subsector con la información que se obtiene al aplicar del método Delphi a los expertos (Astigarraga, 2008; López, 2018), se implementa una matriz de cambio (Mera, 2015), se extraen las variables más relevantes para su posterior calificación de acuerdo con su influencia y dependencia lo que permite alcanzar las variables clave del entorno del sistema. Posteriormente, mediante la utilización del método Mactor (Godet, 2007), se evalúa la importancia y relevancia de los actores más influyentes. Paralelamente, se realiza el análisis morfológico en donde se construyen futuros posibles enfocados en la relación objetiva que tienen las variables de mayor incidencia y los actores más relevantes. Finalmente, se expone un escenario apuesta que puede ser alcanzado a través de la formulación de estrategias o acciones clave de acuerdo con los criterios de importancia y gobernabilidad que proporciona la Matriz IGO (Mojica, 2008).

\section{Dinámica del subsector}

El Municipio de Ipiales está ubicado al sur del Departamento de Nariño con un área de participación territorial del 8,1\%. Dentro de las actividades económicas que generan mayor valor agregado en el municipio se encuentra el subsector comercio con un 22\%. En el año 2019, el total de establecimientos de comercio al por mayor y menor, incluyendo la reparación de automotores y motocicletas, fue de 2406 generando 3720 empleos directos. Es innegable que Ecuador es un socio comercial indispensable para el municipio y el Departamento de Nariño. Esto se puede evidenciar en los porcentajes de exportaciones e importaciones comerciales generadas con el vecino país, las cuales en 2019 fue de 8,3\% (por debajo de Estados Unidos que representó un 20,8\%) y de 61,1\%, respectivamente (Cámara de Comercio de Ipiales, 2020).

Es necesario subrayar que esta relación comercial está expuesta a las variaciones de la tasa de cambio, es decir que a medida que se presenta una devaluación del peso colombiano, la actividad del subsector comercio crece, mientras que, si se presenta una revaluación del peso, el comercio local se reactiva en las ciudades del Ecuador (Terán, 2020). 
Por lo tanto, el subsector comercio adquiere gran importancia en la dinámica económica de la Frontera, posicionándose como su principal actividad. Su fortalecimiento, es indispensable, sobre todo cuando el 50,2\% de los empresarios se vieron en la necesidad de cerrar de manera temporal sus establecimientos de comercio en la época de cuarentena y confinamiento provocada por la pandemia (Cámara de comercio de Ipiales, 2021b).

En Colombia, se han elaborado algunos trabajos que aportan al estado actual de los estudios prospectivos en diferentes sectores, tales como el sector agrícola en el desarrollo de la cadena productiva del plátano en Risaralda (Suarez et al., 2020), el futuro de las empresas cafeteras en el Quindío con implementación de las NIFF (Garcés \& Torres, 2018) y de vigilancia tecnológica en el sector agropecuario en Colombia (Florez \& Uribe, 2018). En el sector industrial, estudios que tienen que ver con el futuro del diseño, confección y comercialización de la moda (Velásquez et al., 2020), distribución eficiente de energía eléctrica (Gómez et al., 2018), futuro del sector metalmecánico (Betancourt \& Cruz, 2018) y desarrollo vehicular (López, 2017) han sido desarrollados. Por otra parte, los estudios prospectivos también han sido relevantes dentro del sector servicios en temas como agua potable y saneamiento básico (Ruiz et al., 2020), seguridad social y salud (Paredes et al., 2019), estrategias para la educación superior (Espinal et al., 2020), desarrollo de Software (Piña et al., 2019), transporte sostenible y transporte marítimo Internacional (Quintero, 2019; Quintero et al., 2018), generación de empresas del sector solidario (Romero et al., 2019) y lo relacionado al futuro de las políticas públicas para el financiamiento de las Pymes en Colombia (Medina, 2019).

\section{Metodología}

\section{Análisis estructural}

El análisis estructural (Dueñas et al., 2019), inicia mediante la aplicación del instrumento de recolección de información matriz de cambio (Mera, 2015) a ocho personas expertas que tienen una relación profesional o de trabajo con el sistema estudiado, esto facilita reconocer variables del entorno. En esta primera parte se logra identificar las variables clave y más influyentes del sistema, además, se fijan estrategias para la evolución y desarrollo del subsector comercio al por mayor y menor de la zona de frontera en el Municipio de Ipiales. 
Se tiene en cuenta la siguiente clasificación:

- Variables Clave: son las variables más importantes que reducen la complejidad del sistema, son las más motrices, de alta influencia y dependencia, se encuentran en la zona superior derecha del plano (Aceituno, 2017).

- Variables Reguladoras: son variables que en el estudio no definen su influencia y dependencia, en cierto momento de incidencia o movimiento del mismo sistema pueden adquirir algún tipo de relevancia, se encuentran en la zona central del plano (Ballesteros \& Ballesteros, 2008).

- Variables Autónomas o excluidas: son variables que se encuentran desconectadas y no son determinantes en el sistema, no ejercen un peso relevante en el estudio, están separadas, pero pueden interactuar en casos muy especiales, se ubican en la zona inferior izquierda del plano (Mendoza et al., 2011).

- Variables Resultado: son variables que dependen de otras que tienen un peso más relevante del sistema, además, son variables dependientes y de baja motricidad, se ubican en la zona inferior derecha del plano (Pérez \& Vargas, 2016).

Para continuar con el análisis estructural e identificar las variables clave se aplicó la herramienta Mic Mac -Matriz de Impacto cruzado aplicada a una clasificación-, su objeto es "identificar las variables más motrices y dependientes, construyendo una tipología de las variables mediante clasificaciones directas e indirectas" (Godet, 2007, p. 84). Con esta herramienta, es posible presentar las variables del sistema subsector comercio en un plano de dos ejes y "observar los resultados de las relaciones evaluadas por los expertos en la calificación de la matriz" (Noguera, 2009, p. 19).

De la información brindada se extrajeron 22 variables que corresponden al objeto de estudio, ellas son: Contrabando en la zona de Frontera (Contra/F), Desempleo (Desempleo), Migración poblacional (Migración), Incentivos comerciales locales (Incentivos), Capacitación a comerciantes (Capacit), Efectos de Pandemia (Pandemia), Costos de transporte (Costostran), Política de ordenamiento de espacio público (Espaciopub), Carga Impositiva (Impuestos), Informalidad en la actividad comercial (Informalid), Brecha Tecnológica (Brechatecn), Tasa de 
cambio (TCN), Valor Agregado (Valoragreg), Precios competitivos (Precios), Inseguridad (Insegurida), Sistema financiero (Sistfinanc), Incertidumbre Política (Incert/Pol), Geoubicación (Geoubicaci), Distribución y comercialización (Dist/comer), Innovación (Innovación), Inflación (Inflación), Gestión Comercial (Gest/Comer).

\section{Análisis de Actores}

Mediante la utilización del método de análisis de actores se buscan las relaciones de convergencia y divergencia entre los actores, sus estrategias, fuerza y posición (Moreno, 2019). Participan en la toma de decisiones influyentes sobre el subsector comercio al por mayor y menor en el Municipio de Ipiales e involucran posturas y objetivos relevantes que se asocian en primera medida al realizar el análisis estructural.

Para realizar el análisis, se utiliza la herramienta Mactor -Matriz de Alianzas y Conflictos, Tácticas, Objetivos y Recomendaciones- (Quinteros \& Hamann, 2017). "El objetivo de la utilización de este método es el de facilitar a un actor una ayuda para la decisión de la puesta en marcha de su política de alianzas y conflictos" (Godet et al., 2000, p. 75).

Se tiene en cuenta la siguiente clasificación:

- Actores de enlace. Poseen niveles altos de influencia y dependencia, son determinantes en la forma en cómo actúa el sistema y tienen una dependencia directa con el mismo (Noguera, 2009).

- Actores Dependientes. Este grupo de actores posee alta dependencia y poca influencia en el sistema (Camelo y Treviño, 2014).

- Actores Autónomos. No influyen en las decisiones del sistema o su relación es muy baja, pueden considerarse que son actores aislados (Garza y Cortez, 2011)

De acuerdo al análisis estructural se identificaron los siguientes actores relevantes del estudio: Oficina de Desarrollo Económico, Dirección de Impuestos y Aduanas Nacionales, Policía Fiscal y Aduanera, Servicio Nacional de Aprendizaje, Cámara de Comercio de Ipiales, Asociación de Comerciantes, Empresarios e Industriales de Ipiales, Academia, Sistema de Investigación, 
Desarrollo Tecnológico e Innovación, Secretaria de Hacienda, Sistema Financiero, Comercio Informal, Gobernación de Nariño, Ministerio de Comercio, Industria y Turismo, Secretaría de Salud.

\section{Análisis Morfológico}

Para Chung (2018), el análisis morfológico está orientado a explorar de manera coherente los futuros posibles, partiendo del estudio de todas las combinaciones de la desagregación de un sistema. Además, este análisis ayuda a construir escenarios posibles y asigna una calificación de acuerdo a la ocurrencia de las variables de mayor probabilidad, su ventaja sobre otros métodos radica en que reduce la incertidumbre, descompone el sistema (Rodríguez et al., 2019) y permite construir y diseñar escenarios detalladamente (Hernández et al., 2020). El estudio morfológico inicia con la construcción de escenarios utilizando las variables clave y las relaciones existentes entre ellas que se obtiene del análisis estructural. Posteriormente, se realiza una reducción del espacio muestral eliminando algunas condiciones de hipótesis que nos son probables en el futuro, dando prioridad a los escenarios de mayor probabilidad y por último combinando las hipótesis que resultan de la reducción de escenarios (Garavito, 2008).

El análisis utilizado en esta investigación, ajustó y relacionó tres hipótesis de futuros posibles con las variables clave dispuestas en el análisis estructural, llevando dicha relación a plantear los escenarios de un sistema, que permitan realizar las estrategias para su cumplimiento (Godet \& Durance, 2007).

\section{Resultados}

\section{Análisis Estructural}

En la Figura 1 se muestra el mapa de influencia y dependencia resultante de la utilización del software especializado Mic Mac, se observan cuatro cuadrantes que definen de manera diferente la posición de las variables y que son presentados de la siguiente manera: 


\section{Figura 1}

\section{Mapa de Influencia y Dependencia}

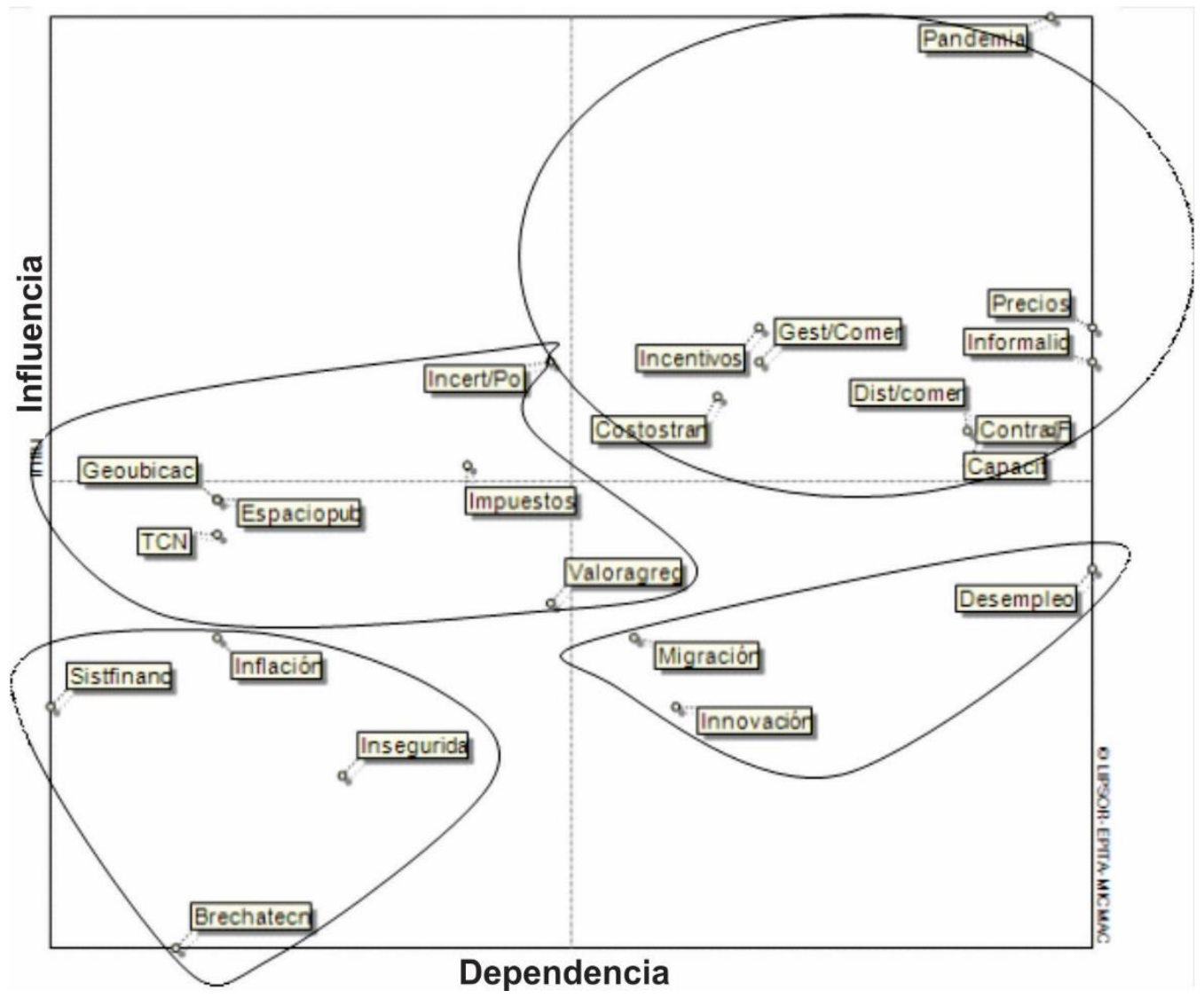

Fuente: elaboración propia.

En la Figura 1 se observan y definen las variables expuestas en el sistema y su importancia en el estudio. Dichas variables son las siguientes:

Variables Clave: Los costos de transporte (Costostran) están determinados por gastos operativos que se presentan en la conectividad vial, costos de insumos (combustible, peajes, llantas entre otros) (Clavijo, 2014) que afectan el mercado de oferta y demanda de bienes y servicios (López y Pardo, 2019). Los incentivos comerciales locales (Incentivos) se detallan como beneficios en estímulos para la inversión local y extranjera cómo mejoras en infraestructura, servicios, entre otros (Barreix \& Velayos, 2021). Gestión Comercial (Gest/comer) es la actividad que administra y ejerce una influencia a un objetivo para lograr el mejor resultado posible (Morales, 2014). Distribución y comercialización (Diste/comer) busca organizar actividades que son convenientes 
para la adquisición de bienes o servicios del subsector (Morales, 2014). Los precios competitivos (precios) se establecen cuando en el mercado existen precios más bajos que los de la competencia. Informalidad en la actividad comercial (informalid) se infiere que es una actividad de la economía informal se lleva a cabo en puestos improvisados en la calle y que genera una actividad de subsistencia (Villa et al., 2020). Contrabando en la zona de frontera (Conra/F): según Muriel \& Acosta (2020) "Consiste en la introducción y extracción del territorio aduanero nacional de mercancías sin pago de los derechos de aduana” (p. 143). Capacitación a comerciantes (Capacit) es un instrumento educativo que genera un valor agregado y se transmite a los interesados el conocimiento y aplicabilidad de nuevos instrumentos y procesos que rediseñen la cadena de suministro (Muriel \& Acosta, 2020). La variable efectos de pandemia (Pandemia) distingue los efectos económicos negativos que ocasiona la pandemia por el virus SARS-CoV-2 (Covid 19) (Cepal, 2020).

Variables Reguladoras: De acuerdo con Garcia et al., (2020), la incertidumbre política (Incert/po) provoca que las empresas retrasen sus decisiones de inversión como una medida de precaución, lo que conlleva a situaciones estáticas y proclives al cambio. Respecto a la variable Carga Impositiva (Impuestos), la disminución de impuestos fomenta la inversión y empleabilidad aunque se corre el riesgo de que la mano de obra sea desplazada por maquinaria (Farné, 2020). Valor agregado (Valoragreg) nace con la mejora de la cadena de valor teniendo en cuenta el importe económico generado en un proceso productivo el cual mide la oportunidad del negocio (Vinajera et al., 2017). Geoubicación (Geoubicac) se refiere a la ubicación geográfica de los establecimientos y empresas comerciales, la cual representa una estrategia adecuada para que las operaciones logísticas faciliten la llegada de productos a los clientes (Naranjo et al., 2018). Política de ordenamiento de espacio público (Espaciopub) es la política pública que configura el espacio público como un lugar definido para el aprovechamiento ciudadano, para su esparcimiento y circulación y no dispuesto a actividades de comercio (Páramo \& Contreras, 2018). Tasa de Cambio Nominal (TCN) se define como una variable relevante en la toma de decisiones en el mercado e indicador de riesgo para la inversión (Catalán, 2021).

Variables Autónomas o excluidas: La variable sistema financiero (Sistfinanc) es la inclusión financiera comercial y empresarial para su financiamiento formal, generación de empleo y 
productividad (Valderrama et al., 2021). Inflación (Inflación) se define como el incremento del índice de precios al consumidor vinculado con el comportamiento de los precios de la canasta familiar (Valderrama et al., 2021). Inseguridad (Insegurida) es la variable que está determinada por la delincuencia social y que afecta el desempeño del comercio (Cepal, 2020). Brecha tecnológica (Brechatecn) es la variable que se presenta cuando existe desigualdad de acceso a la información de la red comercial mediante las nuevas tecnologías (Peña et al., 2017).

Variables Resultado: Migración poblacional (Migración) explica que la llegada de personas de otros países fortalece la informalidad y el desempleo y afecta la estabilidad comercial de la zona de frontera (Ramírez \& Ospina, 2021). Innovación (Innovación) es el empoderamiento de la actividad comercial en el diseño de soluciones productivas (Seclen \& López, 2020) y la variable desempleo (Desempleo) relaciona la falta de oportunidad de la población en la zona de frontera que está en edad de trabajar y que no encuentra ocupación (Villa et al., 2020).

La clasificación de variables fue un proceso que dejó muchas enseñanzas. Ello permitió confirmar la importancia de algunas de ellas, pero de igual manera develó ciertas variables que debido a sus acciones indirectas juegan un papel principal -y que la clasificación directa no pone de manifiesto- (Godet et al., 2000).

En la Figura 2, se representa el plano de influencias directas, "que permite denotar la relación de los componentes del sistema" (Noguera, 2009, p. 28) y el grado de influencia que una variable tiene sobre la otra "de acuerdo a su motricidad y dependencia conforme al sometimiento o impacto que tiene esa relación sobre las demás" (Camelo y Treviño, 2014, p. 125). Se concluye que las variables más importantes son las que poseen mayor relación entre sí y que deben ser tenidas en cuenta en la creación de los escenarios. 


\section{Figura 2}

Plano de influencias directas

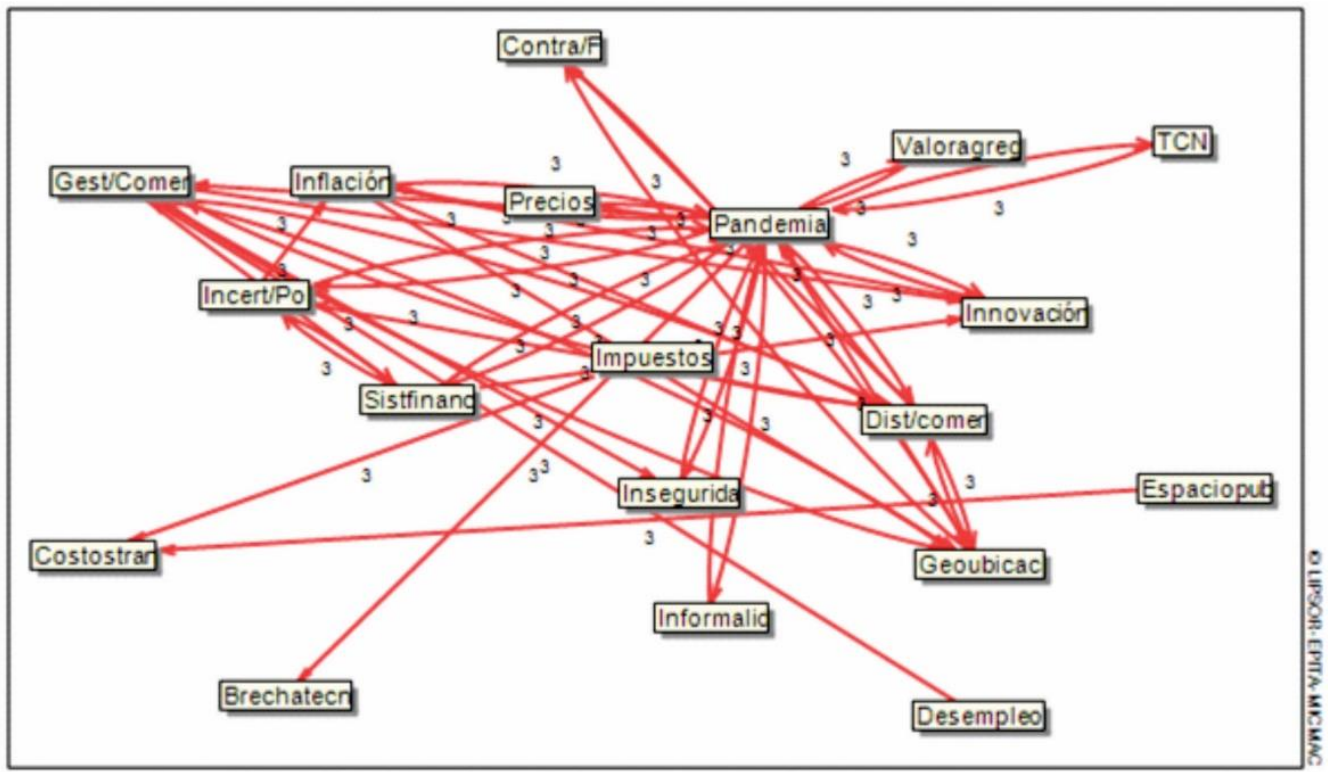

Fuente: elaboración propia.

\section{Análisis de Actores}

Las soluciones planteadas a continuación son una reflexión en donde se identifican posibles conflictos entre actores y su relación con las variables clave expuestas en el análisis estructural. Según Noguera (2009) y Torres (2018), en esta parte se derivan los objetivos estratégicos que se plantean como acciones para llevar a cabo con relación a los problemas identificados.

En la Figura 3 se muestra la información que resulta del análisis de influencia y dependencia entre actores, de la siguiente manera: 


\section{Figura 3}

Plano de influencia y dependencia entre actores

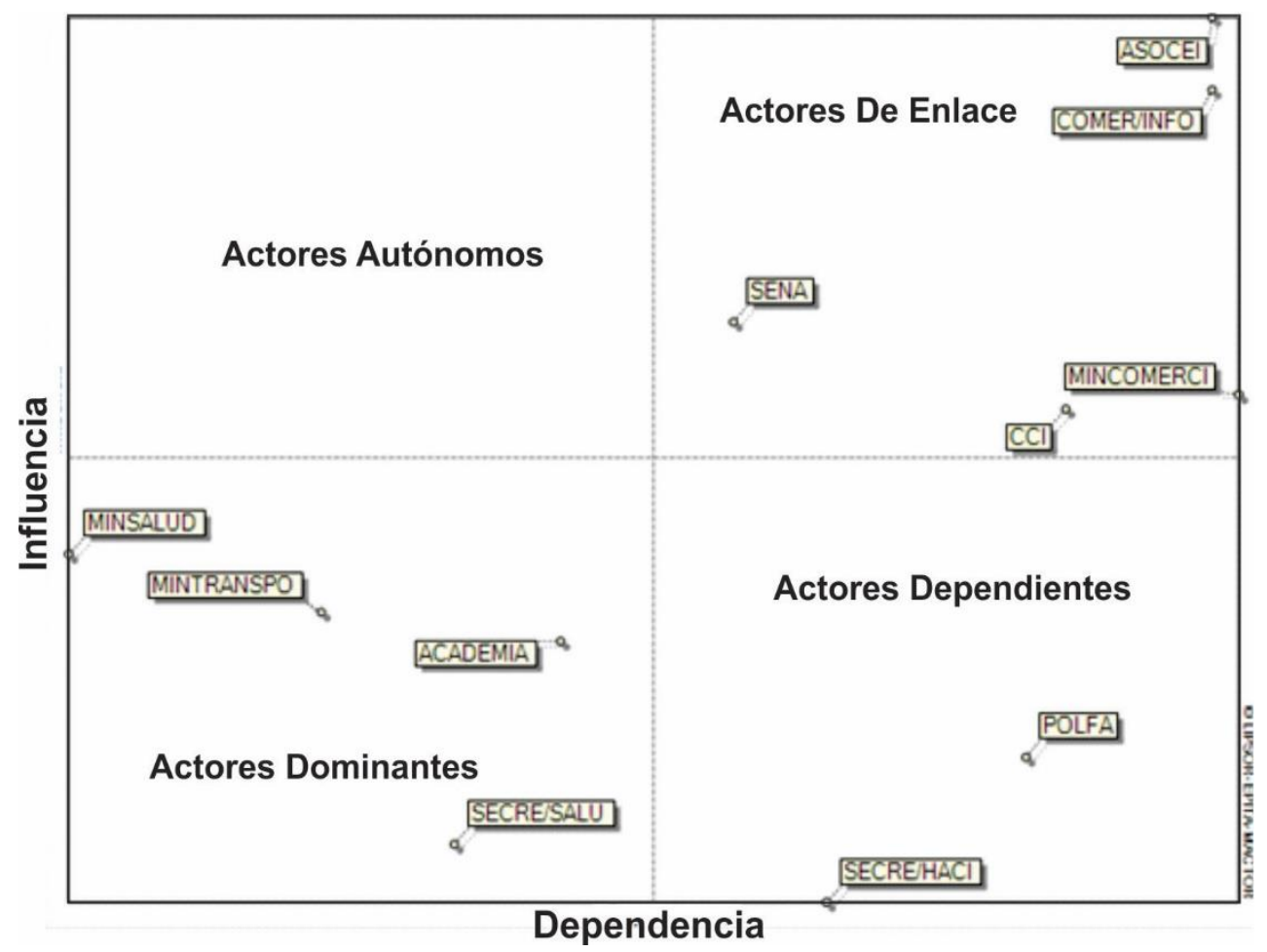

Fuente: elaboración propia.

Una vez se obtuvo el resultado de la relación entre actores, se pudo conocer las actitudes, la relación que poseen y el acuerdo de los objetivos planteados. Los actores de mayor influencia y dependencia son en su orden:

Actores enlace: La Asociación de Comerciantes y Empresarios de Ipiales (ASOCEI) son los, encargados de velar por los derechos y deberes de los comerciantes y empresarios (Cámara de comercio de Ipiales, 2021b). La Cámara de Comercio de Ipiales (CCI) es una entidad encargada de promover la formalización comercial y empresarial y el desarrollo sostenible de la región (Cámara de comercio de Ipiales, 2021a). El comercio informal (COMER/INFO) es definido como un grupo de personas que realizan actividades comerciales y no son reconocidas por ninguna institución (Cámara de comercio de Ipiales, 2021b). El Servicio Nacional de Aprendizaje (SENA) es una entidad del estado colombiano que ofrece formación gratuita en programas técnicos, tecnológicos y complementarios (Servicio Nacional de Aprendizaje, 2021) y 
el Ministerio de Comercio, Industria y Turismo (MINCOMERCIO) apoya la actividad empresarial y actividades turísticas mediante planes, programas y proyectos.

Actores dependientes: La Policía Fiscal y Aduanera (POLFA) es una institución cuya misión es garantizar la seguridad fiscal y apoyar a la Dirección de Impuestos y Aduanas Nacionales DIAN (Dirección de gestión de Policiía fiscal y aduanera, 2021). La Secretaría de Hacienda del Municipio de Ipiales (SECRE/HACI) es la institución que se encarga de administrar los recursos municipales y es la receptora de impuestos comerciales.

Actores dominantes: En este cuadrante se encuentra el Ministerio de Salud y Protección Social (MINSALUD) que se encarga de dirigir, evaluar y orientar el sistema de seguridad social en salud (Ministerio de Salud y Protección Social, 2021). El Ministerio de Transporte (MINTRANSPO) encargado de adoptar políticas, programas y proyectos que relacionen el tránsito, infraestructura y transporte (Ministerio de transporte, 2021). Las instituciones educativas y universidades (ACADEMIA) y la Secretaría de Salud del Municipio de Ipiales (SECRE/SALU).

Para el juego de alianzas y de conflictos que se ponen de manifiesto en el sistema, el método Mactor contribuye a la formulación de preguntas clave o correctas y reducir las incoherencias en la elaboración de los escenarios de la prospectiva (Godet \& Durance, 2009). Por ello, es pertinente que estas preguntas permitan la construcción de hipótesis de futuro del subsector comercio al por mayor y menor en el Municipio de Ipiales; las preguntas son las siguientes:

- ¿Qué tan probable es que para el año 2030 los costos de transporte sean los adecuados para el fortalecimiento del subsector comercio facilitando la distribución, comercialización y el desarrollo competitivo del Municipio de Ipiales?

- ¿Qué tan realizable es que para el año 2030 se cree y organice el clúster de comercio fronterizo que permita la competitividad con estrategias de valor y precio en el subsector comercio del Municipio de Ipiales? 
- ¿Qué tan asequible es que para el año 2030 se permita para el subsector comercio tarifas preferenciales de pago de impuesto de industria y comercio a comerciantes emprendedores como incentivo que evita la informalidad en la actividad comercial del Municipio de Ipiales?

- ¿Qué tan probable es que para el año 2030 se permitan incentivos comerciales como la gestión de subsidios para actividades productivas, gestión de tarifas preferenciales de registro comercial, incremento de campañas efectivas para la formalización y se capacite a los comerciantes en programas técnicos y tecnológicos, actividades e innovación productiva, que influyan en la generación de valor agregado en el Municipio de Ipiales?

- ¿Qué tan probable es que para el año 2030 disminuya el contrabando en la zona de frontera debido al incremento de la competitividad del sector y consumo de productos locales, regionales y nacionales, sin represión de las autoridades?

- ¿Qué tan factible es que para el año 2030 los efectos económicos negativos de la pandemia causados en el subsector comercio en el Municipio de Ipiales originada a finales del 2019 hayan desaparecido permitiendo el mejoramiento de las variables que impulsan el sistema?

\section{Análisis Morfológico}

Se definieron las hipótesis que se consideran escenarios mediante el análisis morfológico y se formularon tres escenarios que corresponden a la combinación de hipótesis de futuro relacionadas en el análisis de actores, mencionando, además, la influencia de las variables clave. Los tres escenarios de estudio son:

Subsector comercio, competitividad estratégica. Los costos de transporte de mercancía disminuyen debido al mejoramiento de la infraestructura vial, todo ello ha permitido influir en el precio de productos básicos de primera necesidad de manera favorable, ha elevado el consumo y fortalecido el intercambio con el Ecuador. Además, la disminución del costo en el traslado de la materia prima que se utiliza para elaborar productos locales mejora la competitividad empresarial. El Clúster de comercio fronterizo genera estrategias de valor y precio, el desarrollo de nuevos aliados clave (empresas) produce conglomerados productivos que giran en torno hacia la industrialización. 
Subsector comercio, gestión productiva. Se permiten tarifas preferenciales de pago de impuesto de industria y comercio a empresarios y comerciantes emprendedores con ello se evita la generación de comercio informal, el gobierno nacional brinda subsidios para actividades productivas, se gestiona ante Confecámaras y la Superintendencia de Industria y Comercio tarifas menores de registro comercial, se capacita a comerciantes en programas técnico y tecnológicos que involucran la generación de herramientas para la innovación productiva.

Subsector comercio, expansión económica. Se disminuye el contrabando en la zona de frontera, debido a las campañas de formalización empresarial y comercial, a su vez, el aumento del consumo de productos locales, regionales y nacionales derivado del mejoramiento competitivo genera mayores fuentes de empleo y estabilidad económica en la zona. Los efectos económicos negativos generados por la pandemia en el subsector comercio han desaparecido debido a la consolidación económica positiva que se viene generando desde que el 90\% de la población recibió la vacuna en contra del nuevo coronavirus SARS-CoV-2 en el Municipio de Ipiales y en la región.

\section{Escenario Apuesta}

En la Tabla 1 se muestra la clasificación de escenarios de acuerdo con Zambrano (2012). El escenario apuesta fue sometido a la calificación de probabilidad de ocurrencia por parte de ocho expertos a quienes se le aplicó el método Delphi, según Godet (2007) y Miklos \& Tello (2007), un escenario es un conjunto de eventos que tiene que presentar una cierta coherencia y describe una situación deseable con una trayectoria de tendencias que permiten pasar de la situación origen a la situación futura. Además, "los escenarios son un ejercicio narrativo que contrasta diferentes realidades en donde caben lo peor que pudiera suceder con lo mejor que pudiera suceder dentro de un sistema de estudio" (Miklos \& Arroyo, 2015, p. 72). 


\section{Tabla 1}

Clasificación de escenarios

\begin{tabular}{cccc}
\hline Escenarios & $\begin{array}{c}\text { Competitividad } \\
\text { estratégica }\end{array}$ & $\begin{array}{c}\text { Gestión } \\
\text { productiva }\end{array}$ & $\begin{array}{c}\text { Expansión } \\
\text { económica }\end{array}$ \\
\hline Expertos & \multicolumn{3}{c}{ Certidumbre } \\
\hline Experto 1 & $80 \%$ & $60 \%$ & $60 \%$ \\
Experto 2 & $75 \%$ & $80 \%$ & $75 \%$ \\
Experto 3 & $90 \%$ & $80 \%$ & $70 \%$ \\
Experto 4 & $70 \%$ & $70 \%$ & $65 \%$ \\
Experto 5 & $80 \%$ & $60 \%$ & $50 \%$ \\
Experto 6 & $95 \%$ & $82 \%$ & $73 \%$ \\
Experto 7 & $92 \%$ & $75 \%$ & $87 \%$ \\
Experto 8 & $78 \%$ & $50 \%$ & $60 \%$ \\
Promedio & $83 \%$ & $70 \%$ & $68 \%$ \\
Desviación estándar & $-0,0695$ & $-0,0690$ & $-0,0691$ \\
Coeficiente de variación & $10,72 \%$ & $16,89 \%$ & $16,78 \%$ \\
\hline
\end{tabular}

Fuente: Fuente: Adaptado de Zambrano (2012).

\section{Estrategias para el escenario Apuesta}

Para determinar lo requerido y efectuar el escenario apuesta, las estrategias fueron vinculantes con los actores del sistema identificados en este estudio y para ello se utilizó la Matriz IGO (Mojica, 2008). De acuerdo con el grado de probabilidad y cumplimiento de los tres escenarios planteados en el sistema, el escenario de mayor probabilidad de ocurrencia en el subsector comercio al año 2030 es competitividad estratégica (Tabla 1).

En la Tabla 2 se muestra el escenario apuesta y la distribución en consenso con los expertos. Según la importancia de las acciones la escala de puntuación fue: 4 (muy importante), 3 (importante), 2 (poco importante), 1 (sin importancia). Respecto a la gobernabilidad de la acción se propuso la calificación de Fuerte (5), Moderada (3), Débil (1) y Nulo (0) (Gándara et al., 2014). 


\section{Tabla 2}

Matriz IGO

\section{Escenario Apuesta del Subsector comercio al por mayor y menor del Municipio de} Ipiales a 2030

Los costos de transporte de mercancía disminuyen debido al mejoramiento de la infraestructura vial, todo a ello ha permitido influir en el precio de productos básicos de primera necesidad de manera favorable, ha elevado el consumo y fortalecido el intercambio con el Ecuador. Además, la disminución del costo en el traslado de la materia prima que se utiliza para elaborar productos locales mejora la competitividad empresarial.

El Clúster de comercio fronterizo genera estrategias de valor y precio, el desarrollo de nuevos aliados clave (empresas) produce conglomerados productivos que giran en torno hacia la industrialización en el Municipio de Ipiales.

\begin{tabular}{|c|c|c|c|c|c|c|}
\hline & & & \multicolumn{4}{|c|}{ Gobernabilidad } \\
\hline No. & Acciones/Estrategias & Importancia & $\mathbf{F}$ & $\mathbf{M}$ & $\mathbf{D}$ & $\mathbf{N}$ \\
\hline 1 & $\begin{array}{l}\text { Rediseñar la cadena de suministro, productores, } \\
\text { proveedores, empresas y comercio que ofrezcan } \\
\text { líneas ventajosas de fletes que permitan reducir } \\
\text { costos. }\end{array}$ & 4 & $\mathrm{X}$ & & & \\
\hline 2 & $\begin{array}{l}\text { Integrar el transporte terrestre y aéreo con el } \\
\text { subsector comercio con una línea directa sin } \\
\text { intermediarios que le permitan bajar costos y se } \\
\text { beneficie el precio final. }\end{array}$ & 3 & & & & $X$ \\
\hline 3 & $\begin{array}{l}\text { Aprovechar la infraestructura vial } 4 \mathrm{G} \text { en el } \\
\text { Departamento de Nariño para el transporte de } \\
\text { mercancías en hacia el Municipio de Ipiales. }\end{array}$ & 4 & & $\mathrm{X}$ & & \\
\hline 4 & $\begin{array}{l}\text { Diseñar, gestionar financiación e implementar } \\
\text { empresas de carácter comercial, como anclas del } \\
\text { desarrollo competitivo en el Municipio de Ipiales. }\end{array}$ & 2 & $\mathrm{X}$ & & & \\
\hline 5 & $\begin{array}{l}\text { Diseñar estrategias que permitan al comercio de la } \\
\text { región conectar con los centros de producción y de } \\
\text { paso abrir la puerta a la inclusión social con } \\
\text { crecimiento económico. }\end{array}$ & 4 & & $\mathrm{X}$ & & \\
\hline 6 & $\begin{array}{l}\text { Fortalecer a la asociación de comerciantes y } \\
\text { empresarios de Ipiales para empoderar su gestión en } \\
\text { robustecer el subsector comercio en capacitaciones } \\
\text { para calificar mano de obra y aprovechamiento de } \\
\text { los costos de producción. }\end{array}$ & 3 & $\mathrm{X}$ & & & \\
\hline 7 & $\begin{array}{l}\text { Diseñar e implementar un proyecto de adaptabilidad } \\
\text { tecnológica para mejorar la logística de } \\
\text { ofrecimiento de productos y publicidad del }\end{array}$ & 2 & & & $\mathrm{X}$ & \\
\hline
\end{tabular}


subsector comercio en el Municipio de Ipiales.

Realizar un diagnóstico del subsector comercio del

Municipio de Ipiales que sirva como insumo para identificar los actores clave que formaran parte del

Clúster comercial de la frontera.

Establecer la cadena de valor que involucre todas

9 las acciones relevantes que impulsen el subsector comercio en el Municipio de Ipiales.

Crear incentivos tributarios que permitan la

10 inversión privada en el comercio formal en el Municipio de Ipiales.

F: fuerte; M: moderada; D: débil; N: nulo.

Fuente: Elaboración propia

Por último, y de acuerdo con los expertos, se resume en la Tabla 3 la utilidad directa de la planeación estratégica en acciones inmediatas, acciones retos y acciones menos urgentes para alcanzar el escenario apuesta del subsector comercio al por mayor y menor en el Municipio de Ipiales a 2030.

\section{Tabla 3}

Clasificación de estrategias para alcanzar el escenario apuesta a 2030

\begin{tabular}{|c|c|}
\hline No. & Estrategias Inmediatas: Acciones muy importantes y gobernadas \\
\hline 1 & $\begin{array}{l}\text { Rediseñar la cadena de suministro, productores, proveedores, empresas y comercio que } \\
\text { ofrezcan líneas ventajosas de fletes que permitan reducir costos. }\end{array}$ \\
\hline 2 & $\begin{array}{l}\text { Aprovechar la infraestructura vial } 4 \mathrm{G} \text { en el Departamento de Nariño para el transporte de } \\
\text { mercancías hacia el Municipio de Ipiales. }\end{array}$ \\
\hline 3 & $\begin{array}{l}\text { Diseñar estrategias que permitan al comercio de la región conectar con los centros de } \\
\text { producción y de paso abrir la puerta a la inclusión social con crecimiento económico. }\end{array}$ \\
\hline 4 & $\begin{array}{l}\text { Fortalecer a la asociación de comerciantes y empresarios de Ipiales para empoderar su } \\
\text { gestión en robustecer el subsector comercio en capacitaciones para calificar mano de } \\
\text { obra y aprovechamiento de los costos de producción. }\end{array}$ \\
\hline 5 & $\begin{array}{l}\text { Realizar un diagnóstico del subsector comercio del Municipio de Ipiales que sirva como } \\
\text { insumo para identificar los actores clave que formarán parte del Clúster comercial de la } \\
\text { frontera. }\end{array}$ \\
\hline 6 & $\begin{array}{l}\text { Establecer la cadena de valor que involucre todas las acciones y actores relevantes que } \\
\text { impulsen el subsector comercio en el Municipio de Ipiales. }\end{array}$ \\
\hline
\end{tabular}

Estrategias Reto: Acciones muy importantes y poco gobernables

1 Integrar el transporte terrestre y aéreo con el subsector comercio con una línea directa sin 
intermediarios que le permitan bajar costos y se beneficie el precio final.

Diseñar e implementar un proyecto de adaptabilidad tecnológica para mejorar la

2 logística de ofrecimiento de productos y publicidad del subsector comercio en el Municipio de Ipiales y la región

3

Crear incentivos tributarios que permitan la inversión privada en el comercio formal en el Municipio de Ipiales.

\section{Estrategia Menos Urgente}

1 Diseñar, gestionar financiación e implementar empresas de carácter comercial, como anclas del desarrollo competitivo del Municipio de Ipiales.

Fuente: Elaboración propia

Los resultados obtenidos corroboran la necesidad de la intervención institucional pública y privada en la implementación de las estrategias y acciones formuladas. Las variables clave que arroja el análisis estructural están apoyadas por las acciones de las variables reguladoras que en cualquier momento pueden ser relevantes en el redireccionamiento estratégico y en estudios posteriores. La incidencia de los actores enlace sobre las estrategias definidas requieren un gran compromiso presupuestal y de capital intelectual, cuya interrelación facilitaría el fortalecimiento del contexto social, político y económico del Subsector Comercio al por mayor y menor en el Municipio de Ipiales. El análisis Morfológico es utilizado en estudios muy relacionados con el subsector comercio como la cadena láctea (Zambrano, 2012), mipymes dedicadas al desarrollo de software (Díaz \& Ospina, 2013), cadena productiva de frutas (Betancourt \& Scarpetta, 2017), entre otros.

Por otra parte, los resultados pueden ampliarse con la utilización de otras herramientas prospectivas que permitirían la creación de escenarios como los ejes de Peter Schwartz (Schwartz \& Medina, 2011) o la herramienta de transformación de futuros (Inayatullah, 2012).

Por último, el escenario apuesta está relacionado con planes, programas y proyectos de instituciones regionales que apoyan la mejora de la gestión de la competitividad del subsector comercio en aspectos muy importantes como el desarrollo de infraestructura, ferias comerciales, fortalecimiento institucional y desarrollo tecnológico, esperando que en el horizonte 2030 se consolide y permita su evolución. a pesar de los cambios de gobierno. 


\section{Conclusiones}

La incidencia que tiene la articulación de los actores que giran en torno al subsector comercio en el municipio de Ipiales determina un futuro alcanzable.

El escenario apuesta posee factores muy importantes que se relacionan con el cambio y que son factibles en el año 2030. Para ello, es necesario implementar las siguientes estrategias: rediseñar la cadena de suministro con ventaja competitiva, aprovechar la nueva infraestructura vial 4G, conectar con los centros de producción directamente, fortalecer la asociatividad, conformar el Clúster comercial de la frontera y establecer la cadena de valor del subsector comercio.

Los grandes retos y que son complemento para lograr el escenario apuesta son: integrar el transporte aéreo con una línea directa que permitan bajar costos y que incida en el precio final, lograr la adaptabilidad tecnológica, crear incentivos tributarios y gestión de financiación ante instituciones locales y nacionales.

La dependencia del subsector comercio en la zona de frontera colombo - ecuatoriana en la generación de empleo y mejoramiento de la calidad de vida de sus habitantes es muy fuerte. La competitividad se logra fijando bases estructurales con redes empresariales e institucionales, con un acompañamiento continuo de organizaciones y equipos multidisciplinarios que coadyuven con la evolución de las estrategias hacia el escenario al año 2030.

\section{Referencias}

(1) Aceituno, P. (2017). Prospectiva: bases y práctica fundamental para la toma de decisiones (Primera ed). Universidad Tecnológica Metropolitana. https://bit.ly/3BA3Y9z

(2) Astigarraga, E. (2008). El método Delphi. Universidad de Deusto. Facultad de CC.EE. y Empresariales este. https://bit.ly/3eWI2f5

(3) Ballesteros, D. \& Ballesteros, P. (2008). Análisis estructural prospectivo aplicado al sistema logístico. Scientia Et Technica, 14, 194-199. https://bit.ly/3i2hQBR

(4) Barreix, A. \& Velayos, F. (2021). Incentivos tributarios, compromisos internacionales y 
suficiencia recaudatoria Otra trilogía imposible. In Banco Interamericano de Desarrollo (IDB-DP-86). https://bit.ly/3j5moYi

(5) Betancourt, B. \& Cruz, J. (2018). Escenarios futuros del sector metalmecánico. Municipio de Tuluá y su zona de influencia. Horizonte 2018-2028. Informador Técnico, 82(2), 181. https://doi.org/10.23850/22565035.1408

(6) Betancourt, B. \& Scarpetta, G. (2017). Prospectiva estratégica de la cadena productiva de frutas y hortalizas del Valle del Cauca. Revista Estratégica Organizacional, 6(2). https://doi.org/https://doi.org/10.22490/25392786.2474

(7) Cámara de comercio de Ipiales. (2020). El comercio exterior en la economía de la ciudad de Ipiales (Nariño), frontera con Ecuador. (Primera ed). Cámara de Comercio de Ipiales. https://bit.ly/3cJuVgl

(8) Cámara de comercio de Ipiales. (2021a). Estudio económico del Municipio de Ipiales - 2021 (Segunda Ed). Cámara de Comercio de Ipiales. https://bit.ly/3cJuVgl

(9) Cámara de comercio de Ipiales. (2021b). Impactos económicos y empresariales por el Covid19, en la jurisdicción de la cámara de comercio de Ipiales. Investigaciones Económicas, 23. https://bit.ly/3cJuVgl

(10) Camelo, G. y Treviño, A. (2014). El análisis estructural. In G. Gandara \& F. Javier Osorio (Eds.), Métodos prospectivos: Manual para el estudio y la construcción de futuro (Primera ed, pp. 121-141). PAIDOS.

(11) Catalán, H. (2021). Fundamentales macroeconómicos del tipo de cambio. Evidencia de cointegración. Cuaderno de Economía, 40(83), 557-582. https://doi.org/https://doi.org/10.15446/cuad.econ.v40n83.82607

(12) Cepal. (2020). América Latina y el Caribe ante la pandemia del COVID-19. Efectos económicos y sociales. Informe Especial Covid-19, 1-15. https://bit.ly/3DLUyIv

(13) Chung Pinzas, A. R. (2018). Teoría y práctica de la prospectiva (Primera ed). Alfaomega.

(14) Clavijo, S. (2014). Costos de Transporte, Multimodalismo y la competitividad de Colombia. https://bit.ly/3p1ZODs

(15) Díaz, M. \& Ospina, M. (2013). Prospectiva 2014 - 2018 para mipymes dedicadas al desarrollo de software por encargo en Colombia. Informador Técnico, 77(1), 69-85. https://doi.org/https://doi.org/10.23850/22565035.46

(16) Dirección de gestión de Policía fiscal y aduanera. (2021). Quienes somos. 
https://bit.ly/2YZlx41

(17) Dueñas, J., Medina, A., Ramírez, L., Camacho, W. \& Sobenis, J. (2019). La prospectiva estratégica como herramienta de planeación a largo plazo. Magazine de Las Ciencias, 4(3), 1-18. https://bit.ly/3kS7ztz

(18) Espinal, D., Scarpetta, G. \& Cruz, N. (2020). Análisis prospectivo estratégico de la educación superior en Colombia. Cultura Educación Y Sociedad, 11(1), 177-196. https://doi.org/10.17981/cultedusoc.11.1.2020.13

(19) Farné, S. (2020). ¿Menos impuestos a las empresas, más empleo formal? El caso de Colombia. Revista Instituto Colombiano de Derecho Tributario, 83, 1-24. https://bit.ly/3p4ptf4

(20) Florez, D. \& Uribe, C. (2018). Diseño metodológico para la integración de herramientas de prospectiva y vigilancia tecnológica en la definición de lineamientos estratégicos de ciencia, tecnología e innovación para el sector agropecuario en Colombia. In M. Cuervo \& F. Guerrero (Eds.), Prospectiva en América Latina: Aprendizajes a partir de la práctica (Primera ed, Vol. 88, p. 237). CEPAL. https://bit.ly/3DNt0m5

(21) Gándara, G., Leal, A. \& Palomino, C. (2014). Matriz FODA y matriz IGO. In G. Gándara \& F. Osorio (Eds.), Métodos prospectivos: Manual para el estudio y la construcción de futuro (Primera ed, 223-338). PAIDOS.

(22) Garavito, J. (2008). Aplicación de análisis estructural y morfológico a los factores determinantes para la seguridad en Bogotá [Universidad de los Andes]. https://bit.ly/3vcNtNS

(23) Garcés, L. \& Torres, M. (2018). Prospectiva del sector cafetero del Quindío con la implementación de las NIIF. In M. Redondo, A. Barrera, \& C. Duque (Eds.), Internacionalización de la investigación en las ciencias sociales (Primera ed, 122-147). Universidad Libre Seccional Pereira. https://bit.ly/2YJbU9z

(24) García, B., García, L. \& Gómez, R. (2020). ¿La incertidumbre política afecta a la inversión en el Ibex 35? Cuaderno de Economía, 43(122), 163-174. https://doi.org/https://doi.org/10.32826/cude.v42i122.102

(25) Garza, J. y Cortez, D. (2011). El uso del método MICMAC y MACTOR análisis prospectivo en un área operativa para la búsqueda de la excelencia operativa a través del Lean Manufacturing. Innovaciones de Negocios, 8(16), 335-356. https://bit.ly/3BOYBDI 
(26) Godet, M. (2007). De la anticipación a la acción manual de prospectiva y estrategia (1 edición). Marcombo. https://bit.ly/35mY0da

(27) Godet, M. \& Durance, P. (2007). Prospectiva estratégica métodos y problemas (Segunda ed). Instituto Europeo de Prospectiva y Estrategia. https://bit.ly/3rvPtz3

(28) Godet, M. \& Durance, P. (2009). La prospectiva estratégica para las empresas y los territorios (Primera ed, Vol. 10). Laboratoire d'Innovation de Prospective Stratégique et d'Organisation. https://bit.ly/2WmtGON

(29) Godet, M., Monti, R., Meunier, F. \& Roubelat, F. (2000). Caja de herramientas de prospectiva (Primera ed). Cuaderno de Lipsor. https://bit.ly/3xpbCk2

(30) Gómez, J., Sandoval, C. \& Coronel, J. (2018). Análisis de prospectiva del sector energético de Colombia para la integración de fuentes fotovoltaicas en los sistemas de distribución de energía eléctrica aplicando a una revisión en base de datos científicas. Revista Colombiana de Tecnologías de Avanzada, 2(32), 109-119. https://doi.org/https://doi.org/10.24054/16927257.v32.n32.2018.3034

(31) Grabendorff, W. (2017). Relaciones vecinales y gobernanza regional: un desafío para la región Andina. In C. Guzman \& L. Trejos (Eds.), Más allá de las fronteras: integración, vecindad y gobernanza: Colombia, Ecuador, Perú (Primera ed, 1-21). Universidad del Norte.

(32) Hernández, Y., Rodriguez, M. y Espinosa, M. (2020). Planeación estratégica prospectiva en las organizaciones turísticas. Alfa Publicaciones, $1(3), \quad 20-38$. https://doi.org/10.33262/ap.v1i3.4

(33) Inayatullah, S. (2012). El pensamiento prospectivo como herramienta de transformación. In B. Eric \& M. Guilló (Eds.), Prospectiva e innovación Vol. 1 (primera ed, 113-154). Plaza y Valdés.

(34) López, C. y Pardo, S. (2019). El transporte de carga terrestre en el comercio internacional. Análisis comparativo entre Bogotá, Colombia y Santa Cruz de la Sierra, Bolivia. Ensayos de Economía, 29(54), 89-114. https://doi.org/10.15446/ede.v29n54.75022

(35) López, Carlos. (2017). Identificación de escenarios prospectivos y líneas de acción para el desarrollo del sector de motocicletas colombiano. Espacios, 38(22), 1-15.

(36) López, E. (2018). El método Delphi en la investigación actual en educación: Una revisión teórica y metodológica. Educación XX1. 21(1), 17-40. https://bit.ly/3i3fsKX 
(37) Medina, J. (2019). Prospectiva de las políticas gubernamentales del modelo de financiación en el crecimiento de las Pymes en Colombia. Contexto, 8, 18-34. https://doi.org/10.18634/ctxj.8v.0i.980

(38) Mendoza, A., Quintero, I. \& Coronado, E. (2011). Aplicación de Técnicas Prospectivas. Ingeniare, 11, 25-36.

(39) Mera, C. (2015). Pensamiento prospectivo: visión sistémica de la construcción del futuro. Análisis, 46(84), 89-104. https://bit.ly/3iMA4pR

(40) Miklos, T. \& Arroyo, M. (2015). El oficio prospectivo en la práctica. In B. Paz (Ed.), Planeación prospectiva estratégica: Teorías, Metodologías y buenas prácticas en América latina (Primera Ed, pp. 69-92). Metadata. https://bit.ly/3gtAuR0

(41) Miklos, T. \& Tello, M. E. (2007). Planeación prospectiva: una estrategia para el diseño de futuro ('Primera e). Limusa.

(42) Ministerio de Salud y Protección Social. (2021). Institucional. https://bit.ly/3FNrnqd

(43) Ministerio de transporte. (2021). ¿Quiénes Somos? https://bit.ly/2YPP2oM

(44) Mojica, F. (2008). La construcción del futuro: concepto y modelos de prospectiva estratégica, territorial y tecnológica (Primera re). Universidad Externado de Colombia.

(45) Morales, Á. (2014). Gestión Comercial "Un desafio constante en un mundo globalizado" (Primera ed). Centro de Marketing Ltda.

(46) Moreno, A. (2019). Software como herramienta para métodos de prospectiva estratégica: MICMAC. In J. Mejía (Ed.), Análisis multivariante con enfoque dependiente en las ciencias de la administración como base para la innovación (Primera ed, 51-59). Universidad de Guadalajara. https://bit.ly/2V6V2I0

(47) Mosquera, L. y Forero, V. (2017). Análisis de los factores que inciden en la dinámica empresarial de las ciudades de Ipiales y Tulcán (2010-2014). SATHIRI - Sembrador, 12, 33-47. https://doi.org/10.32645/13906925.33

(48) Muriel, J. \& Acosta, J. (2020). La subfacturación de mercancías como expresión del delito de contrabando y sus efectos en Colombia para 2016. Revista CIES, 11(2), 141-159. https://bit.ly/3j31SK5

(49) Naranjo, R., Zabala, H., Castellanos, G., Gómez, M. \& Hernández, N. (2018). Geoposicionamiento logístico de tiendas de la Economía Popular y Solidaria en Riobamba. Ingeniería Industrial, 34(1), 101-112. https://bit.ly/2YNLd3J 
(50) Noguera, Á. (2009). Enseñando prospectiva (Primera Ed). Universidad Colegio Mayor de Nuestra Señora del Rosario. https://bit.ly/3goW2PX

(51) Páramo, P. \& Contreras, M. (2018). Formación ciudadana en comportamientos urbanos responsables: Etnografía rápida orientada a la convivencia en el espacio público urbano. Cuadernos de Vivienda y Urbanismo, 11(22), 1-17. https://doi.org/https://doi.org/10.11144/Javeriana.cvu11-22.fccu

(52) Paredes, A., Marín, F., Martínez, K., Inciarte, A. \& Luque, L. (2019). Retos del sistema general de seguridad social y salud: prospectiva para el desarrollo social en la Costa Atlántico de Colombia. Revista Cubana de Salud Pública, 45(1), 1-22.

(53) Peña, H., Cuartas, K. \& Tarazona, G. (2017). La brecha digital en Colombia: Un análisis de las políticas gubernamentales para su disminución. Redes de Ingeniería, O(0), 59-71. https://doi.org/10.14483/2248762X.12477

(54) Pérez, R. \& Vargas, H. (2016). El uso del método MICMAC, para la definición de procesos de intervención en las organizaciones. Ciencia y Poder Aéreo, 11(1), 92-105.

(55) Piña, J., Castaño, D., Hernández, L. \& Garro, J. (2019). Análisis prospectivo de la industria de desarrollo de software en Colombia. Punto de Vista, 10(16), 5-24. https://doi.org/10.15765/pdv.v11i16.1415

(56) Quintero, J. (2019). Desarrollo Orientado al Transporte Sostenible (DOTS). Una prospectiva para Colombia. Bitácora Urbano Territorial, 29(3), 59-68. https://doi.org/10.15446/bitacora.v29n3.65979

(57) Quintero, J., Ramírez, Y. \& Cortázar, A. (2018). Transporte marítimo internacional: aspectos operativos y comercio, logística portuaria, aspectos jurídicos y comerciales, prospectiva para Colombia. Journal de Ciencia e Ingeniería, 10(1), 18-31. https://bit.ly/3aAG9SV

(58) Quinteros, J. \& Hamann, A. (2017). Planeamiento estratégico prospectivo: Métodos Mactor y Smic (Primera ed). Ecoe Ediciones Ltda.

(59) Ramírez, J. \& Ospina, M. (2021). La política migratoria en Colombia y Ecuador: decisiones y respuestas a la migración venezolana. In L. Nejamkis, L. Conti, \& M. Akskal (Eds.), (Re) pensando el vínculo entre migración y crisis. Perspectivas desde América Latina y Europa (Primera ed, p. 343). CLACSO. https://bit.ly/3mU41qd

(60) Rodríguez, K., Nicolás, S. y Ramos, E. (2019). La relación banca - empresa estatal: estudio 
de casos en el sector del turismo. Cofin Habana, 13(1), 1-13. https://bit.ly/31GN0jW

(61) Romero, C., De la Puente, K. \& Alfonso, L. (2019). Diagnóstico sobre la prospectiva de generación de empresas y proyectos productivos de emprendimiento social y economías creativas en el sector solidario. IN Crescendo, 10(1), 223-243. https://doi.org/10.21895/incres.2019.v10n1.14

(62) Ruiz, C., Salazar, D. \& Rodríguez, N. (2020). La prestación de los servicios de agua potable y saneamiento básico en Colombia análisis y prospectiva. In Documentos FCE-CID, Escuela de Economía (34). https://bit.ly/2YMABBZ

(63) Schwartz, P. \& Medina, J. (2011). La planificación estratégica por escenarios. Cuadernos de Administración, 14(21), 199-225. https://doi.org/10.25100/cdea.v14i21.114

(64) Seclen, J. \& López, H. (2020). Influencia del uso de herramientas en la gestión de la fase temprana del proceso de innovación de productos. Innovar, 30(76), 119-130. https://doi.org/10.15446/innovar.v30n76.85217

(65) Servicio Nacional de Aprendizaje. (2021). Quienes somos. https://bit.ly/3DF3tva

(66) Solarte, M., Solarte, C. \& Rivera, G. (2020). Emprendimiento frontera colombo ecuatoriana en el escenario del post acuerdo. In J. Martínez (Ed.), Avances en investigación científica. Tomo II. Ciencias económicas y sociales (Primera ed, 123-142). Corporación Universitaria Autónoma de Nariño.

(67) Suarez, L., Escobar, N.,\& Zartha, J. (2020). Estudio de prospectiva al 2032 de la cadena de plátano, un enfoque hacia los programas académicos del sector agroindustrial. Información Tecnológica, 31(6), 95-104. https://doi.org/10.4067/S0718-07642020000600095

(68) Terán, G. (2020). Análisis de la problemática de frontera colombo ecuatoriana por medio de análisis no estructurado. Visión Empresarial, 10, 53-69. https://doi.org/10.32645/13906852.1009

(69) Torres, V. (2018). Teoría y práctica de la prospectiva estratégica en América Latina (Primera ed). Pontificia Universidad Católica del Ecuador.

(70) Valderrama, J., Medina, F., Peña, C., Castro, F. \& Londoño, D. (2021). Inclusión financiera empresarial: evidencia con registros administrativos (No. 526; Archivos de Economía). https://bit.ly/3p4WM1w

(71) Velásquez, S., Giraldo, D. \& Botero, L. (2020). Análisis de escenarios futuros como método prospectivo para la Mesa Sectorial de Diseño, Confección y Moda en Colombia. Revista 
Espacios, 41(21), 375-390.

(72) Villa, V., Ayaviri, D., Velarde, R., Quispe, G. \& Cruz, J. (2020). La informalidad en el comercio, caracterización económica, laboral, administrativa y tributaria. Revista Inclusiones, 7(2), 309-330. https://bit.ly/3DEKAZi

(73) Vinajera, A., Marrero, F. \& Ruiz, M. (2017). Método para calcular el valor agregado en cadenas de suministro de productos electromecánicos. Revista Chilena de Ingeniería, 25(3), 535-546. https://bit.ly/3iYTlW8

(74) Zambrano, F. (2012). Prospectiva de la cadena láctea del Departamento de Nariño al horizonte del año 2020. Tendencias, XIII (1), 36-54. https://bit.ly/3idVvS1

Cómo citar este artículo: Chalapud, E. (2021). Prospectiva estratégica del subsector comercio del municipio de Ipiales - Colombia, hacia el año 2030. Tendencias, 23(1), 139-166. https://doi.org/10.22267/rtend.222301.186 